\title{
Relative Rates of Some Very Rapid Gaseous Bimolecular Reactions*
}

\author{
Jerome Daent and R. A. Marcus \\ Department of Chemistry, Polytechnic Institute of Brooklyn, Brooklyn, New York
}

(Received March 26, 1956)

\begin{abstract}
The present paper describes a steady-state flow apparatus which permits the measurement of relative rates of rapid gas phase bimolecular association reactions at very low pressures. Using this apparatus, the relative reactivities of the methylamines with boron trifluoride were determined over the pressure range 0.009 to $0.04 \mathrm{~mm} \mathrm{Hg}$. Within the experimental error the ratio of rate constants, $k \mathrm{CH}_{3} \mathrm{NH}_{2} / k\left(\mathrm{CH}_{3}\right)_{3} \mathrm{~N}$, was found to be pressure independent and to have a value of $1 / 12$. A small number of experiments was made competing monomethylamine and dimethylamine for $\mathrm{BF}_{3}$; for this system the ratio of rate constants, $k \mathrm{CH}_{3} \mathrm{NH}_{2}$ / $k\left(\mathrm{CH}_{3}\right)_{2} \mathrm{NH}$ had an average value of $1 / 5$. In addition to the above, experiments were made on various phenomena such as the sorption of $\mathbf{B F}_{3}$ on various surfaces, the selectivity of a diffusion pump, and the extent of base exchange reactions, insofar as these factors pertained to the understanding of the relative rate measurements.
\end{abstract}

\section{INTRODUCTION}

$\mathrm{D}$ URING recent years there has been a revival of experimental and theoretical interest in unimolecular reactions and bimolecular association processes. Several recent theories have been proposed to supplement the earlier formulations ${ }^{1}$ of Hinshelwood, Rice and Ramsperger, and Kassel. These more recent approaches ${ }^{2,3}$ make assumptions concerning the nature of the activated state and concerning the degrees of freedom which play a role in the reaction. On the basis of these assumptions and with the aid of spectroscopic data and the energy of activation of the unimolecular reaction, they can be used to calculate the rate constants of unimolecular reactions and of bimolecular association reactions as a function of the pressure. Another theory related to one of these $\mathrm{e}^{3}$ has recently been proposed. ${ }^{4}$

Most of the reactions which were formerly believed to be simple unimolecular processes are now known to have, at least in part, complex chain reaction mechanisms. Some of the data now regarded as legitimate examples of simple unimolecular reactions and bimolecular association reactions have been summarized in several recent texts. ${ }^{5}$ There are many data on the

* This research is abstracted from a thesis submitted by Jerome Daen to the Graduate Faculty of the Polytechnic Institute of Brooklyn in partial fulfillment of the requirements for the degree of Doctor of Philosophy in Chemistry, June, 1955. A portion of this research was supported by the Office of Naval Research, Contract Nonr 839(09).

$\dagger$ Du Pont Predoctoral Fellow: 1952-1953; Present address: Institute for Fluid Dynamics and Applied Mathematics, University of Maryland, College Park, Maryland.

i See L. Kassel, The Kinetics of Homogeneous Gas Reactions (Chemical Catalog Company, Inc., New York, 1932).

2 N. B. Slater, Proc. Roy. Soc. (London) A194, 112 (1948); Trans. Roy. Soc. A246, 57 (1953).

${ }^{3}$ R. A. Marcus and O. K. Rice, J. Phys. \& Colloid Chem. 55, 894 (1951); R. A. Marcus, J. Chem. Phys. 20, 359 (1952).

4 Rosenstock, Wallenstein, Wahrhaftig, and Eyring, Proc. Natl. Acad. Sci. 38, 667 (1952).

${ }^{5}$ See A. A. Frost and R. G. Pearson, Kinetics and Mechanism (John Wiley and Sons, Inc., New York, 1953); E. W. R. Steacie, Atomic and Free Radical Reactions (Reinhold Publishing Corporation, New York, 1954), second edition; A. F. Trotman-Dickenson, Gas Kinetics (Academic Press, Inc., New York, 1955). high pressure values of the rate constants and relatively few on their dependence on pressure. The former type of data gives direct information about the activated complex, whether it is "loose" or "rigid," while the latter type of data gives (when used in conjunction with the former) information about the degrees of freedom that readily contribute their energy to a breaking bond or, conversely, accept energy from a bond which is being formed. ${ }^{3}$

Of particular interest to the theory of unimolecular dissociation and bimolecular association processes is the relative behavior of dissociating molecules which have the same strength of the breaking bond but which differ in their number of degrees of freedom. This problem can also be studied by measurements of the reverse process of bimolecular associations using a series of molecules of different number of degrees of freedom. The association of various amines with boron trifluoride, a very rapid reaction, provides an interesting example of such a system.

As a source of data for very fast reactions, several methods having potentially wide applicability can be suggested. One, which has been used previously, is an ingenious modification of the Polanyi diffusion flame technique and was devised by Kistiakowsky and coworkers. ${ }^{6}$ A second method involves a competitive steady state system and forms the basis of the present paper. In the former, one reagent is allowed to diffuse into a slowly moving atmosphere of another reagent; a knowledge of the steady state temperature distribution, which is established by a balance of the heat of reaction and the heat losses, permits the rate constant to be estimated. The competitive steady-state flow method measures relative rates by allowing two or more reagents to react with a less than stoichiometric amount of another. Over and above its intrinsic interest, the addition reaction of boron trifluoride with various methylamines was studied to permit a comparison of the results of the competitive flow apparatus with

${ }^{6}$ (a) D. Garvin and G. B. Kistiakowsky, J. Chem. Phys. 20, 105 (1952). (b) G. B. Kistiakowsky and R. Williams, J. Chem. Phys. 23, 334 (1955). 
those of Kistiakowsky et al. A comparison of the results obtained in studies on these association reactions by two very different methods also provides information which should prove useful in studying the validity of the assumptions employed in each technique.

In order to obtain data of inlerest to unimolecular theory, it is usually desirable to work at pressures of the order of $10^{-3} \mathrm{~mm} \mathrm{Hg}$ to $10 \mathrm{~mm}$, since a pressure dependence of these rate constants is generally displayed in this range. Another advantage of low pressure studies, when the relative rates of very fast reactions are measured, lies in the reduction of ambiguities due to the possible influence of local concentration depletion. This point will be discussed in a later section of this paper.

At first, it appeared that a static system would be simpler than a flow system. However, for low pressure work it was found that a steady state flow system offered considerable advantages. For example, the flow system permitted the accumulation of relatively large amounts of residual exit gases, thus simplifying the chemical analysis. Again, considering the relatively small quantities of gas present at low pressures in a static system, it is apparent that in such a system sorption effects can interfere markedly with the chemical analysis.

Prior to a discussion of the apparatus used for measuring the pressure dependence of the relative rates, a summary of background experiments performed will be presented. These included studies on the sorption of $\mathrm{BF}_{3}$ on various surfaces, the development of a suitable analytical technique for mixtures of the methylamines, a verification of the stoichiometry of the reactions, the extent of base displacement reactions, and the pressure and composition dependence of the pumping speed.

\section{EXPERIMENTAL}

\section{Reagents}

All gaseous reagents used in this study were obtained from cylinders of gas sold by the Matheson Company. The specified purities of the compounds used are: monomethylamine- $96.5 \%$, dimethylamine- $98.2 \%$, trimethylamine- $97.0 \%$, and boron trifluoride- $97.0 \%$. (In each of the first three reagents, the impurities are small amounts of the other methylamines and water vapor; in the boron trifluoride, the impurities are air, $\mathrm{SO}_{2}$, and possibly $\mathrm{SiF}_{4}$ ). These compounds were stored in the vacuum system after the removal of noncondensable materials achieved by repeated cycles of freezing at liquid nitrogen temperatures, evacuating the region above the solid followed by melting of the solid. Possible traces of water vapor were removed from the amines by distillation from one trap maintained at the temperature of dry-ice acetone mixtures into another receiver immersed in liquid nitrogen.

Rigorous purification of the reagents was not felt to be needed in these preliminary experiments designed to develop a method for studying relative rates.

\section{Sorption Studies}

The sorption behavior of $\mathrm{BF}_{3}$ and of the amines upon various surfaces was investigated by admitting a known amount of gas into the capillary of a gas buret whose bulb was filled with mercury, and then depressing the mercury level so that the surface of the bulb of the buret was exposed to the gas. The amount of gas used was such that the pressure in the bulb of the buret was about $10^{-3} \mathrm{~mm} \mathrm{Hg}$. After various times, the mercury level was raised so that the gas was once more compressed into the capillary. This procedure was followed for untreated Pyrex walls, methylchlorosilane (General Electric SC 87 waterproofing) treated walls, polythene covered walls, and walls upon which the compound $\left(\mathrm{CH}_{3}\right)_{3} \mathrm{~N}: \mathrm{BF}_{3}$ was deposited. It was found ${ }^{7}$ that the sorption of boron trifluoride on untreated Pyrex glass was appreciable even at low pressures. For example, at pressures of the order of $0.02 \mathrm{~mm}$ boron trifluoride the sorption on glass appeared to be approximately monomolecular. ${ }^{8}$ The sorption on glass walls covered with the various materials listed previously was also found ${ }^{7}$ to be appreciable, though perhaps less than on uncovered Pyrex walls. In each case much of the sorption occurred within several minutes but no detailed rate study was made. Amines were found to react rapidly with much of the sorbed boron trifluoride.

\section{Analytical Method}

Though several other procedures were explored, infrared analysis proved to be the most satisfactory method for mixtures of the methylamines. Spectra of the various gases which had been treated as described were taken on a Perkin-Elmer double beam recording infrared spectrometer model 21, at a series of pressures. To obtain the desired extinction coefficients, the optical densities obtained were divided by the pressure of the gas. Beer's law was found to be obeyed over the pressure range of interest and the average deviations of the coefficients obtained was of the order of $2 \%$. The values were, however, somewhat sensitive to the previous treatment of the reagent since these presumably contained small amounts of the other methylamines. Control analyses to check the reliability of the method were run on several synthetic ternary mixtures; the average deviation of the mole fractions was found to be $\pm 2 \%$.

\section{Stoichiometry}

Though the stoichiometry of the reactions involving the addition of the amines to boron trifluoride is fairly

\footnotetext{
${ }^{7}$ Jerome Daen, Ph.D. thesis, Polytechnic Institute of Brooklyn (1955). This thesis is available on microfilm from University Microfilms, 313 North First Street, Ann Arbor, Michigan.

${ }_{8}$ Unpublished results obtained in this laboratory by E. Helfand and $A$. Zweig.
} 


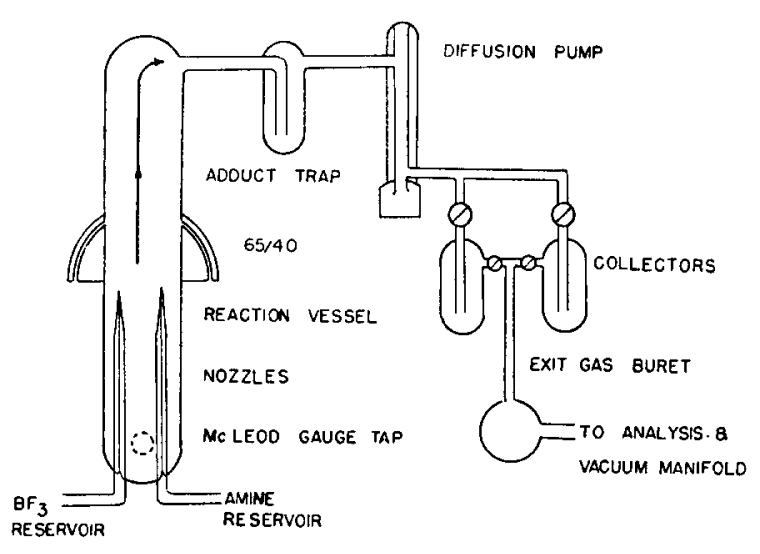

FIg. 1. Schematic diagram of competitive flow apparatus.

well established, several independent studies were made in this laboratory. Known amounts of amine and boron trifluoride were distilled into a vessel and allowed to react; the excess gas was distilled from the white adduct and measured. Within the experimental error, the stoichiometry was found to be one to one. ${ }^{7}$ An alternative procedure would have involved replacing the final transfer by a simple measurement of the pressure of the residual gas in the reaction vessel. However, since in some cases an excess of dimethylamine appeared to "wet" the solid, this later procedure could give erroneous results. When a mixture of solid addition compounds was exposed to dimethylamine vapor the solid appeared to "wet"; however, the removal of the excess amine was always possible even though distillation appeared somewhat slower than usual.

\section{Exchange Reactions}

Some experiments were performed to determine whether any displacement of the base from the adduct occurs when amine is placed in contact with the addition compounds of $\mathrm{BF}_{3}$ and other amines. A measured amount of gaseous amine mixture, a like sample of which was retained for analysis, was placed in contact with the solid addition compounds for fifteen minutes. The gaseous material was subsequently distilled from the vessel containing the solid, analyzed, and compared with the composition of the original mixture. Within the experimental error, no exchange was observed. ${ }^{7}$

\section{Reaction Vessel and Procedure}

The following discussion refers to the diagram of the apparatus given in Fig. 1.

Boron trifluoride and an amine mixture, each in separate storage vessels, were admitted to a reaction space continuously by way of pinhole nozzles. The excess unreacted amines were removed steadily at the top of the reaction vessel, by means of a mercury diffusion pump which was backed by a collector trap immersed in liquid nitrogen. A McLeod gauge attached to the

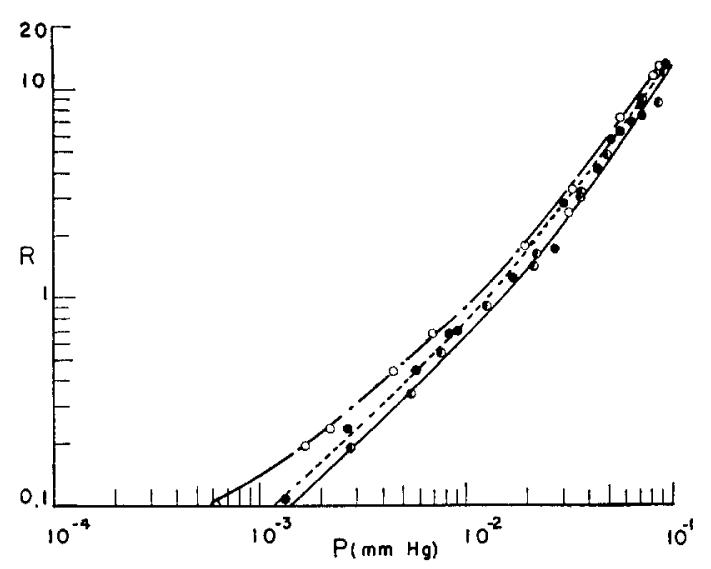

FIG. 2. Pumping speed 2 pressure. $R$ is the rate of flow of gas in $\mathrm{mm} / \mathrm{min}$ into a standard volume of $60.6 \mathrm{cc} . P$ is the stationary pressure in the reaction vessel. $O=$ monomethylamine, $\bullet=$ dimethylamine, $\mathbf{O}=$ trimethylamine.

reaction vessel was used to measure the stationary pressure. After each experiment the collected excess amines were distilled into a standard volume, measured, and distilled for infrared analysis into a Pyrex-walled gas cell equipped with parallel sodium chloride windows.

The influx rate of either reagent into the reaction vessel could be varied by adjusting the pressure in that reagent's storage volume. The storage volumes were made large in comparison to the amount of gas withdrawn to insure that the driving pressure, and consequently the admission rate, remained sensibly constant during an experiment; pressure drops in these volumes were held to a few percent and the driving pressure in each storage volume was generally between 300 and $600 \mathrm{~mm}$, depending on the stationary pressure desired in the reaction vessel.

The reaction vessel was cylindrical in cross section and had an internal diameter of $40 \mathrm{~mm}$ and a length of $290 \mathrm{~mm}$. It was constructed from a $65 / 40$ ball and socket joint to permit easy disassembly for cleaning. Two nozzles were sealed into the lower (ball) end of the unit, as in Fig. 1, while at the upper extremity a two stage mercury diffusion pump maintained the steady state by continuously removing the excess gases. A dry ice-acetone trap was placed before the pump to remove any solid product which might conceivably be carried along by the exit gases and thus prevent possible breakdown and exchange in the hot mercury stream. No mechanical mixing device was used because of its doubtful utility at these low pressures.

In order to interpret the rate data, some knowledge was needed of the dependence of the pumping speed on the stationary pressure in the reaction vessel. In a pumping speed study, the amine storage vessel was first filled to a known pressure with the amine. After evacuation of the reaction assembly to a pressure of $10^{-5} \mathrm{~mm}$ a dry ice-acetone slurry was placed about the adduct trap and liquid nitrogen about each of the dual 
TABLE I. Kinetic data for the reaction of $\mathrm{BF}_{3}$ with the methylamines. ${ }^{\mathrm{a}}$

\begin{tabular}{|c|c|c|c|c|c|c|c|c|c|c|}
\hline Expt. & $R_{1^{0}}$ & $R_{1}$ & $R_{2}$ & $R_{2}$ & $R_{8}{ }^{0}$ & $R_{8}$ & $k_{1} / k_{3}$ & $k_{3} / k$ & $\begin{array}{c}P 0 \times 100 \\
(\mathrm{~mm})\end{array}$ & $P \underset{(\mathrm{mm})}{P \times 100}$ \\
\hline $\begin{array}{r}3 \\
\mathbf{B} \\
\mathbf{5} \\
6 \\
7 \\
8 \\
9 \\
10 \\
11 \\
12 \\
13 \\
14 \\
15 \\
17 \\
18 \\
3 \\
4 \\
5 \\
6 \\
7 \\
1 \\
3 \\
4 \\
2 \\
3 \\
4 \\
5 \\
6 \\
7\end{array}$ & $\begin{array}{l}5.49 \\
7.16 \\
6.26 \\
6.05 \\
4.60 \\
3.20 \\
3.97 \\
3.97 \\
3.52 \\
3.32 \\
3.06 \\
1.52 \\
1.74 \\
1.83 \\
1.63 \\
7.48 \\
7.80 \\
7.49 \\
6.13 \\
4.91 \\
4.85 \\
2.67 \\
3.80 \\
5.34 \\
4.32 \\
4.38 \\
4.05 \\
3.10 \\
2.76\end{array}$ & $\begin{array}{l}4.76 \\
5.79 \\
5.72 \\
5.16 \\
2.92 \\
1.66 \\
3.36 \\
3.39 \\
2.53 \\
2.84 \\
1.85 \\
0.80 \\
0.64 \\
1.05 \\
0.60 \\
5.91 \\
6.86 \\
5.78 \\
3.91 \\
2.73 \\
4.18 \\
1.06 \\
3.40 \\
4.35 \\
3.32 \\
3.81 \\
3.77 \\
2.47 \\
1.78\end{array}$ & $\begin{array}{l}2.84 \\
3.88 \\
3.67 \\
3.35 \\
2.15 \\
1.91 \\
3.03 \\
3.14 \\
2.28 \\
2.41 \\
1.75 \\
1.63 \\
0.75 \\
0.85 \\
0.44 \\
1.77 \\
2.55 \\
2.11 \\
1.81 \\
1.20 \\
1.79 \\
0.68 \\
1.02\end{array}$ & $\begin{array}{l}1.14 \\
2.52 \\
1.81 \\
1.31 \\
0.67 \\
0.41 \\
1.28 \\
1.47 \\
0.93 \\
0.81 \\
0.67 \\
0.35 \\
0.19 \\
0.33 \\
0.11 \\
1.23 \\
1.19 \\
0.86 \\
0.63 \\
0.29 \\
0.79 \\
0.18 \\
0.71\end{array}$ & $\begin{array}{l}3.38 \\
4.95 \\
4.27 \\
4.10 \\
3.08 \\
2.19 \\
3.80 \\
3.69 \\
3.19 \\
3.30 \\
2.69 \\
1.54 \\
1.68 \\
1.88 \\
1.26 \\
8.55 \\
8.95 \\
7.30 \\
6.05 \\
5.19 \\
4.96 \\
2.64 \\
4.05 \\
7.86 \\
8.00 \\
6.80 \\
6.35 \\
5.56 \\
3.99\end{array}$ & $\begin{array}{l}0.91 \\
2.08 \\
1.47 \\
1.23 \\
0.41 \\
0.18 \\
1.22 \\
0.99 \\
0.55 \\
0.75 \\
0.36 \\
0.37 \\
0.080 \\
0.17 \\
0.06 \\
3.26 \\
3.45 \\
2.36 \\
1.36 \\
0.71 \\
1.72 \\
0.26 \\
1.59 \\
3.10 \\
1.70 \\
2.03 \\
2.77 \\
0.69 \\
0.54\end{array}$ & $\begin{array}{l}0.06 \\
0.17 \\
0.05 \\
0.07 \\
0.09 \\
0.08 \\
0.09 \\
0.06 \\
0.08 \\
0.05 \\
0.10 \\
0.28 \\
0.09 \\
0.07 \\
0.09 \\
0.16 \\
0.09 \\
0.14 \\
0.17 \\
0.13 \\
0.09 \\
0.17 \\
0.12 \\
0.15 \\
0.08 \\
0.06 \\
0.05 \\
0.04 \\
0.09\end{array}$ & $\begin{array}{l}0.55 \\
0.39 \\
0.54 \\
0.67 \\
0.34 \\
0.32 \\
0.65 \\
0.42 \\
0.30 \\
0.58 \\
0.25 \\
1.16 \\
0.15 \\
0.16 \\
0.15 \\
0.27 \\
0.71 \\
0.69 \\
0.54 \\
0.50 \\
0.67 \\
0.30 \\
0.28\end{array}$ & $\begin{array}{r}7.7 \\
9.9 \\
9.3 \\
8.9 \\
7.3 \\
5.8 \\
7.5 \\
7.3 \\
6.1 \\
6.3 \\
5.7 \\
4.0 \\
3.7 \\
4.2 \\
\\
9.5 \\
10.8 \\
8.8 \\
7.7 \\
6.3 \\
8.4 \\
5.0 \\
6.8 \\
9.0 \\
8.2 \\
7.3 \\
7.6 \\
6.5 \\
5.8\end{array}$ & $\begin{array}{l}5.3 \\
7.6 \\
6.6 \\
6.7 \\
4.2 \\
2.2 \\
5.4 \\
5.1 \\
3.9 \\
4.2 \\
3.1 \\
1.4 \\
0.87 \\
1.4 \\
0.9 \mathrm{~b} \\
6.8 \\
7.5 \\
5.7 \\
4.2 \\
1.9 \\
5.8 \\
1.9 \\
5.2 \\
6.2 \\
4.7 \\
5.2 \\
5.6 \\
3.0 \\
2.1\end{array}$ \\
\hline
\end{tabular}

$A R$ is the rate of flow of gas in $\mathrm{mm} / \mathrm{min}$, as measured in a standard volume of $60.6 \mathrm{cc}$. The subscripts $1,2,3$ designate mono-, di- and $t r i$-methylamine. $R$ is the rate of flow of gas in $\mathrm{mm} / \mathrm{min}$, as measured in a standard volume of 60.6 cc. The subscripts $1,2,3$ designate mono-, di- and $t r i$-methylamine.
$P$ indicates the stationary pressure in the reaction vessel. In each case the superscript ${ }^{0}$ indicates the flow rates and stationary pressure prevailing before the boron trifluoride has been admitted to the reaction vessel.

b Pressure estimated from flow rate using Fig. 3.

collectors. Dual collectors were employed to eliminate errors due to nonstabilized operating conditions; one was used to establish the steady state and the other to collect the excess amine under steady state conditions. Before an experiment, the pressure in the reaction zone was observed for a 15 or $20 \mathrm{~min}$ period. If there was no increase in pressure, the system was considered leak-free. One collector was then opened to the diffusion pump, which was always operated with a constant heat input. Amine was admitted through the nozzle into the reaction vessel and the pressures in this and in the amine storage vessel noted. The reaction vessel pressure rose initially as time increased. When it became steady for an interval of more than two minutes, the collector in operation was isolated from the diffusion pump and, simultaneously, the other collector was opened. A switch-over of collectors normally took less than five seconds, an interval negligible compared to the total collection time in a run. By the device of allowing a stabilization period, errors due to nonsteady state operation were reduced to a point where they became completely unimportant. After sufficient time had elapsed to insure the collection of an accurately measurable amount of amine, the collector trap was isolated, as was the amine storage vessel. All of the collected amine was distilled, using liquid nitrogen, into a calibrated volume. Warming the solid to room temperature, and noting its pressure in the calibrated volume gave the total collected. The flow rate was determined by dividing this quantity by the time of collection.

Additional points on the speed-pressure curves were obtained by repeating this procedure, the flow rate being varied either by changing the amine storage vessel pressure or the diameter of the pin-point nozzle. In this manner the curves for each of the three methylamines were determined. These results are given in Fig. 2.

The speed pressure behavior of the apparatus towards mixtures of amines was also determined. The procedure here was the same as that for the pure compounds, with the sole exception of the replacement of a single amine by a mixture. The data are included in Table I. In this table the total rate of collection of amines for a given experiment whose stationary pressure in the reaction vessel is $P^{0}$ may be found by summing the values of $R_{1}{ }^{0}, R_{2}{ }^{0}$, and $R_{3}{ }^{0}$ for this experiment. Using a part of the data in this table, this total flow rate is plotted vs $P^{0}$ in Fig. 3, the points being indicated by open circles.

The procedure outlined for the speed-composition experiments also formed the basis for the relative reaction rate experiments. Each of these consisted of two stages. In the first, only amine was passed through the reaction vessel. This yielded the flow rate and the 


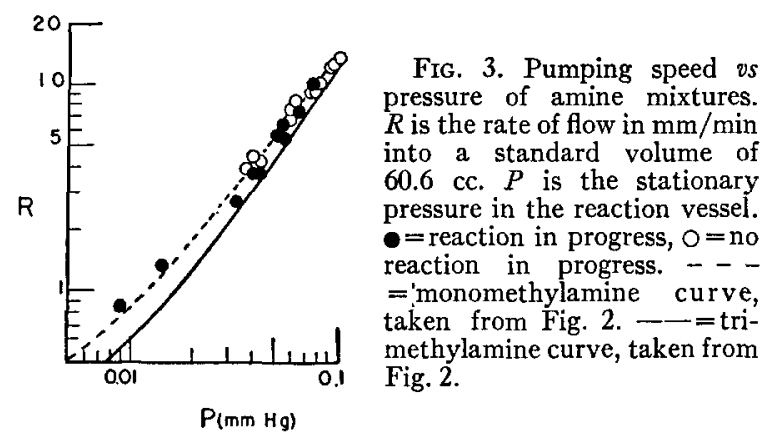

composition of the mixture in the absence of reaction. In the second stage, amines and boron trifluoride were simultaneously passed through the reaction vessel, and the excess unreacted amines were collected. This provided the flow rates and composition of the excess amines after reaction. The influence of geometry was studied in several series of runs that differed only in the distance of the nozzles from the bottom of the vessel and the diffusion pump. These series are identified by a letter prefix, the prefixes having the meanings:

$B$ Series: Both nozzles in the same horizontal plane, $135 \mathrm{~mm}$ above McLeod gauge tap and $150 \mathrm{~mm}$ below top of reaction vessel; ternary mixtures of amines of varying composition and conversion ratio.

$C$ Series: Amine nozzle $135 \mathrm{~mm}$ above the McLeod gauge tap, $\mathrm{BF}_{3}$ nozzle $60 \mathrm{~mm}$ below amine nozzle; ternary mixtures of varying composition and conversion ratio.

$D$ Series: Boron trifluoride nozzle $135 \mathrm{~mm}$ above McLeod gauge tap; amine nozzle $60 \mathrm{~mm}$ below $\mathrm{BF}_{3}$ nozzle; ternary mixtures of varying composition and conversion ratio.

$E$ Series: Boron triffuoride nozzle $135 \mathrm{~mm}$ above McLeod gauge tap; amine nozzle $60 \mathrm{~mm}$ below $\mathrm{BF}_{3}$ nozzle; binary mixtures of monomethylamine and trimethylamine of varying composition and conversion ratio.

The bulk of the reaction zone, as evidenced by the deposition of the solid adduct on the walls, was above the $\mathrm{BF}_{3}$ nozzle. The material precipitated in a relatively rapid manner, none appearing to form beyond, say $50 \mathrm{~mm}$ of that nozzle tip. In the $\mathrm{C}$ series, this reaction zone was between the two nozzles. Since long prismatic needles of mixed adduct were apparent in all experiments, it appeared that the adducts were precipitating out on material already deposited.

Using the above techniques, an attempt was also made to study the reaction of $\mathrm{BF}_{3}$ with ammonia. However, in all trials, the nozzle rapidly clogged with

- As mentioned earlier, this composition, defined by the ratios $R_{1}{ }^{0} / R_{2}{ }^{0} / R_{3}{ }^{0}$, is the composition of the mixture after it has been issued from the nozzle, rather than the composition in the amine driving bulb. The two compositions will differ somewhat because of fractionation of the amines at the nozzle opening, but this is irrel evant to our calculations, since the composition in the amine driving bulb does not appear in them. reaction product. Related observations were first reported by Garvin and Kistiakowsky. ${ }^{6}$ Similarly, when very high percentage conversions of amines were attempted, or when glass wool was packed into the reaction vessel to increase the surface area, rapid nozzle clogging resulted. Under these conditions, of course, no data could be taken. Current experiments are directed towards the elimination of this clogging. An indication of the stability of the system during a run can be obtained from the observation that the stationary system pressures, as given by the McLeod gauge, usually decreased by about $2 \%$. Finally, because of the low activation energy, no attempt was made to thermostat the reaction vessel. The reactions were investigated at room temperature.

The data are presented in Table I, which gives the flow rates of the amines out of the reaction vessel and the corresponding stationary pressure there, both in the presence and in the absence of reaction. The flow rate of boron trifluoride into the reaction vessel during each experiment may be found from Table I, being equal to $\left(R_{1}{ }^{0}+R_{2}{ }^{0}+R_{3}{ }^{0}-R_{1}-R_{2}-R_{3}\right)$. The total flow rate of amines out of the vessel while reaction is in progress, $\left(R_{1}+R_{2}+R_{3}\right)$, is plotted $v s$ the stationary pressure $P$ in Fig. 3 (closed circles), using some of the data in the table.

\section{DISCUSSION}

\section{General}

Two general schemes can be used for relative rate studies: flow systems and static systems. However, certain criteria must be met in both in order to obtain meaningful results. The competitors must be present in excess; the stoichiometry must be clearly defined; no displacement reactions should occur between the reaction products and the excess reagents; there is no local depletion of any competitor during the reaction. Several of these factors can be controlled in a simple manner, and the influence of some others can be conveniently studied. For example, the attainment of an excess of the competing reagents is easily achieved. It has been mentioned previously that no exchange reactions between amines and $\mathrm{BF}_{3}$ amine "salts" were found to occur under conditions of high pressure; since the pressures of each experiment are several orders of magnitude smaller, no exchange would be expected here.

One problem which could arise in the study of the relative rates of very fast reactions is that of local depletion; it is possible that the ratio of concentrations of the two competitors in the reaction zone is not the same as it is outside the zone. In the reaction zone the more reactive competitor will tend to react first and, unless diffusion into the zone is sufficiently rapid, it will tend to become more depleted there than will the less reactive competitor. The effect of this phenomenon, if it occurs, is to make both competitors appear about equally reactive. It will be more important at high 
pressures, where diffusion into the zone is slower. This possible behavior provided an additional reason for investigating these reactions at low pressures. It is encouraging therefore that reactivity ratios very different from unity were obtained.

\section{Selectivity Factors for Pumping}

The composition-pressure speed studies were used to obtain information on the possible existence of different pumping selectivity towards various gases. From Fig. 2 it can be seen that the pump is not highly selective, the ratio of the pumping rates at a given pressure being of the order of unity. It may be estimated from this figure that at low pressures the ratio of conductance of the amines is about $F_{1} / F_{3}=1.43$ and $F_{2} / F_{3}=1.16$, while at high pressures the corresponding quantities are about 1.13 and 1.04. ( $F$, the conductance, is defined as the rate of flow in volumetric units at the prevailing pressure per unit of pressure difference.) If at low pressures the flow rate is limited by collisions with the wall ( $K$ nudsen region), then in this region we would have ${ }^{10}$ $F_{2} / F_{1}=\left(M_{1} / M_{2}\right)^{\frac{1}{2}}$. It would then follow that at low pressures $F_{1} / F_{3}=1.37$ and $F_{2} / F_{3}=1.21$, in good agreement with observed values. At higher pressures where collisions in the gas phase are important there would be some tendency for the conductance to be governed by the Poiseuille equation, ${ }^{10}$ from which it would follow that $F_{2} / F_{1}=\left(M_{1} / M_{2}\right)^{\frac{1}{2}}\left(D_{2} / D_{1}\right)^{2}$, the $D^{\prime}$ s denoting molecular diameters. Because of this second factor $D_{2} / D_{1}$, the conductance ratio at higher pressures should, in the present case, be smaller than that found at lower pressures, as observed experimentally.

However, it should be added that another, much faster two-stage diffusion pump was tested and this one showed an appreciable selectivity toward the amines. ${ }^{7}$ The reason for this behavior is not quite clear but has been discussed elsewhere. ${ }^{7}$ The desirability of choosing

TABLE II. Relative rate constants $\left(k_{1} / k_{3}\right)$ of the reaction of $\mathrm{BF}_{3}$ with $\mathrm{CH}_{3} \mathrm{NH}_{2}$ and $\left(\mathrm{CH}_{3}\right)_{3} \mathrm{~N}\left(R_{1}^{0} / R_{1}>1.25\right)$.

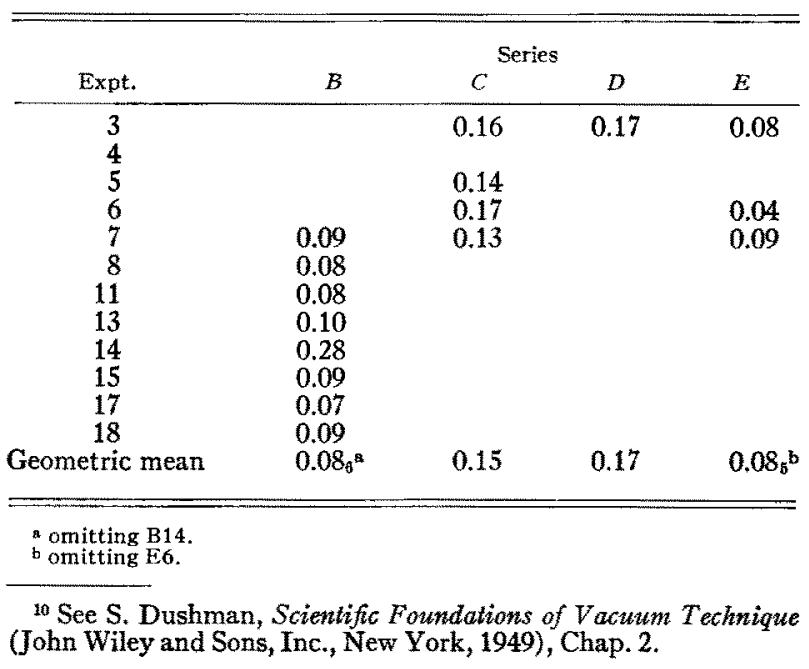

TABLE III. Relative rate constants $\left(k_{2} / k_{3}\right)$ of the reaction of $\mathrm{BF}_{3}$ with $\left(\mathrm{CH}_{3}\right)_{2} \mathrm{NH}$ and $\left(\mathrm{CH}_{3}\right)_{3} \mathrm{~N}$. $\left(R_{2}{ }^{0} / R_{2}<2.5\right.$.

\begin{tabular}{cccc}
\hline & \multicolumn{3}{c}{ Series } \\
Expt. & $B$ & $C$ & $D$ \\
\hline 1 & 0.55 & 0.27 & 0.67 \\
3 & 0.39 & 0.71 & 0.28 \\
4 & 0.54 & 0.69 & \\
5 & 0.65 & & \\
9 & 0.42 & & \\
10 & 0.30 & & \\
11 & 0.46 & $0.70^{\mathrm{a}}$ & 0.43
\end{tabular}

Omitting $\mathrm{C} 3$.

a system with a minimum of selectivity cannot be overemphasized.

Some recent experiments performed in this laboratory indicate that under the present conditions the liquid air trap collector itself has a high pumping efficiency and that the diffusion pump may be serving partly as an orifice.

\section{Rate Equation}

As mentioned previously, the reaction zone is centered somewhat downstream from the boron trifluoride nozzle. There would be expected to be only a very small over-all pressure gradient from one end of the very large diameter reaction vessel to the other. This can be shown to be in agreement with the fact that superimposable exit flow rate-stationary pressure curves were obtained in the presence and absence of boron trifuoride (see Fig. 3).

When the boron trifluoride nozzle is between the amine nozzle and the pump, or at least very near it, then the amines more or less flow through the boron trifluoride zone. Letting $R_{1}{ }^{0}$ represent the rate of flow of the first amine into the system $R_{1}$ its rate of removal by the pump and $k_{1} A_{1} B$ its rate of removal per unit volume by reaction, then an over-all material balance gives

$$
R_{1}^{0}=R_{1}+\int k_{1} A_{1} B d V
$$

where $A_{1}$ and $B$ denote the amine and boron trifluoride pressure, respectively. Similarly for the second amine,

$$
R_{2}{ }^{0}=R_{2}+\int k_{2} A_{2} B d V
$$

Assuming that the amine mixture does not bypass the boron trifluoride zone and that there is no local depletion of the amines, $A_{1}$ and $A_{2}$ in Eqs. (1) and (2) are constant throughout the reaction volume and may be placed outside the integral sign. Furthermore, when selectivity factors are neglected the ratio $A_{1} / A_{2}$ is simply equal to the exit flow rates of the two amines $R_{1} / R_{2}$ during the reaction period. Computing from Eqs. (1) and (2) the ratio of amounts of amines reacted, 


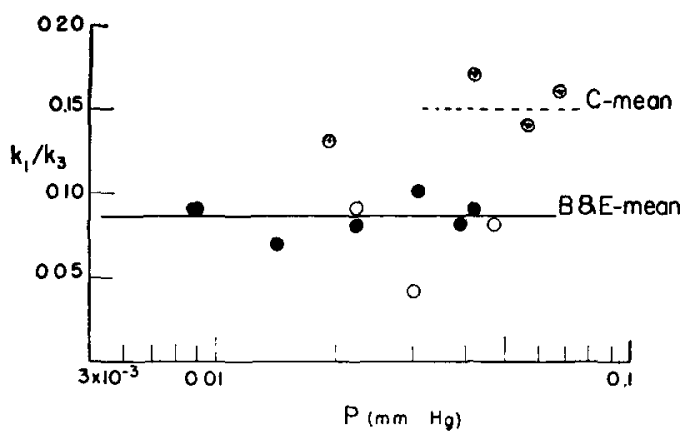

FIG. 4. Ratio of rate constants $k_{1} / k_{3}$ at various pressures, $P$. $\bullet=B$ series, $\odot=C$ series, $O=E$ series.

$\left(R_{1}{ }^{0}-R_{1}\right) /\left(R_{2}{ }^{0}-R_{2}\right)$, and introducing these simplifications, one obtains the relation

$$
\left(R_{1}{ }^{0} / R_{1}-1\right) /\left(R_{2}{ }^{0} / R_{2}-1\right)=k_{1} / k_{2}
$$

Relatively small errors in the flow rates can cause much larger errors in the relative rate constants when $R_{1}{ }^{0} / R_{1} \cong 1$. For the rate constant $k_{1} / k_{3}$ the most important errors arise from the relatively low conversion of the monomethylamine. To eliminate from consideration those values with very low precision, a break point of $R_{1}{ }^{0} / R_{1}=1.25$ was selected, all data having values below this being omitted. A similar discussion applies for the rate constant $k_{2} / k_{3}$ but here a different criterion was applied. In this instance, the conversion ratio of both amines was relatively large and hence was not the controlling error. On the other hand, interferences in analysis were significant when $R_{2}{ }^{0} / R_{2}$ was large. As a result, a break point was chosen so that $R_{2}{ }^{0} / R_{2}$ did not exceed 2.5. Using these two criteria, Tables II and III and Figs. 4 and 5 were obtained.

In computing the mean value of these ratios it was desirable to use a mean which has the following property: The mean for the experimental values for the ratio $k_{1} / k_{3}$, say, should be the reciprocal of the mean of the values for the ratio $k_{3} / k_{1}$. The arithmetic mean does not have this property but the geometric mean does, the geometric mean of a series of measurements $x_{1}, x_{2} \cdots x_{n}$ being $\left(x_{1} x_{2} \cdots x_{n}\right)^{1 / n}$. Accordingly the geometric mean was employed.

Several features of interest are apparent. The average deviations of the relative rate constants are of the order of $20 \%$, which is close to the estimated experimental error; there is no apparent pressure effect in the range of the study. The effect of several nozzle arrangements

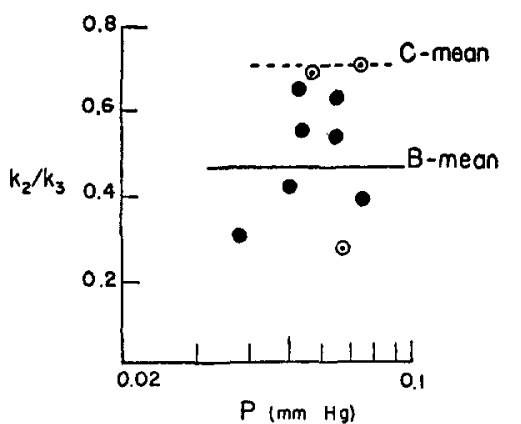

Frg. 5. Ratio of rate constants $k_{2} / k_{3}$ at various pressures, $P$. $\bullet=B$ series, $\odot$ $=C$ series.

upon the data may be inferred from Figs. 2 and 3. It is seen that the results obtained for the ratio of rate constants is closer to unity when the amine nozzle is between the boron trifluoride nozzle and the pump. Perhaps such an arrangement permits some of the amines to bypass the boron trifluoride zone thus tending to make both amines appear more similar in their reactivity.

Over the pressure range studied it is inferred from the data of series $B$ and $E$ that the relative rate constants have the values

$$
k_{1}: k_{2}: k_{3}=1: 5: 12 \text {. }
$$

Kistiakowsky and Williams ${ }^{6}$ give for the limiting low pressure rates

$$
k_{1}: k_{2}: k_{3}=1: 4: 25
$$

while in a more accurate region at $0.1 \mathrm{~mm}$, their equations lead to the relation

$$
k_{1}: k_{2}: k_{3}=1: 5: 8 \text {. }
$$

This agreement between both experimental methods is surprisingly good. However, it is somewhat incomplete since no definite pressure dependence was observed with the competitive flow apparatus for the monotri methylamine combination whereas Kistiakowsky and co-workers' results indicate that one should be present. However, the data of the latter are rather widely scattered, and large errors are possible in the extrapolated rates, particularly for the trimethylamineboron trifluoride reaction. It is also possible that the wall of the reaction vessel serves as a "third body" at the low pressures used in our experiments.

At present work is being done to extend these measurements to higher pressures and to investigate the importance of local depletions in more detail. 